\title{
ASPECTOS CLÍNICOS, SOCIAIS E TRABALHISTAS DA DOENÇA DE CHAGAS EM ÁREA ENDÊMICA SOB CONTROLE DO ESTADO DE MINAS GERAIS, BRASIL
}

\author{
João Carlos Pinto Dias
}

\begin{abstract}
Candidatos a emprego em construção civil na região de Bambuí, MG, submeteram-se a sorologia para doença de Chagas, eletrocardiograma e Rx de tórax e esôfago. De 301 candidatos recentes uma prevalência de $11,6 \%$ de infecção chagásica foi detectada, sendo $5,0 \%$ no grupo etário de 18-29 anos, $13,8 \%$ no de $30-39$ e $39,4 \%$ no de 40 anos $e$ acima. $A$ ocorrência de ECGs alterados foi de $31,3 \%$ no grupo soropositivo contra $7,1 \%$ nos soronegativos $(p<0,05)$ enquanto o aumento de área cardiaca foi de $8,6 \%$ e de $1,5 \%(p<0,05)$ e alteração no trânsito esofágico de $8,6 \% \mathrm{e} 0,8 \%(p<0,05)$ respectivamente. Chagásicos e nãochagásicos provinham das mesmas regiões, sendo os grupos comparáveis quanto a origem rural, morte súbita na família, doação e recepção de sangue. O grupo chagásico apresentou significativamente maior proporção de individuos com antecedentes de trabalho na lavoura $(48,6 \%$ contra $19,5 \%)$ e de individuos analfabetos ou com primário incompleto $(43,8 \%$ contra $19,5 \%)$, frente aos não-chagásicos. Os detalhamentos da pesquisa indicam que o contínuo controle do vetor na região tem sido extremamente eficaz, com impacto sobre a prevalência e a morbidade da esquizotripanose. Por outro lado, indicam que os mecanismos transfusional e congênito de transmissão do Trypanosoma cruzi ao homem continuam a apresentar importância mínima na área pesquisada.
\end{abstract}

Palavras-chaves: Doença de Chagas. Trabalho. Morbidade. Controle.

Os estudos sobre os aspectos clínicos e médicosociais da tripanosomose americana têm sido da maior importância na definição política do controle da doença em suas áreas de dispersão ${ }^{2} 6$. Estabelecidas em números gerais a prevalência $\mathrm{e} a$ distribuição da endemia ao longo de praticamente 18 países latinoamericanos, do Méxicoà Patagônia, a questão básica para as autoridades sanitárias se fixa na importância da infecção chagásica em termos sociais e de custo benefício, uma vez que seu controle é perfeitamente exeqüivel frente aos principais mecanismos de transmissão do Trypanosoma cruzi ao homem ${ }^{81722}$. Em verdade, os dados de morbi-mortalidade da doença de Chagas, embora disponíveis para algumas regiões, apresentam-se insuficientes ou inconclusivos para a maioria dos países endêmicos, disto resultando

\footnotetext{
Departamento de Clínica Médica da Faculdade de Medicina da Universidade Federal de Minas Gerais, Belo Horizonte, MG. Endereço para correspondência: Dr. João Carlos Pinto Dias. Depto. de Clínica Médica. Centro de Pesquisas René Rachou/ FIOCRUZ. Caixa Postal: 1743, 30161-970 Belo Horizonte, MG, Brasil. Fax: (031) 295-3115.

Recebido para publicação em $12 / 02 / 93$.
}

(entre outras razões) a falta de programas contínuos e rotineiros de controle em pelo menos 13 dos países afetados $^{822}$. Com a evolução histórica e políticosocial desses países, não apenas a nova tendência da tripanosomose como endemia urbana, mas também o peso do chagásico como integrante da força de trabalho vem chamando a atenção dos pesquisadores ${ }^{11}$ 1619 .

Emparticular, preocupa o problema do indivíduo infectado que permanece praticamente incógnito e desassistido nas áreas endêmicas, muitas vezes já acometido por formas iniciais da cardiopatia chagásica crônica e a merecer uma assistência médico-social $\operatorname{minima}^{7}{ }^{10}$.

O presente estudo foi realizado no Centro Emmanuel Dias, em Bambuí, Minas Gerais, região outrora altamente endêmica para doença de Chagas, mas desde 1974 sob rigoroso controle de transmissão 5 . Objetivou-se avaliar o impacto da infecção sobre trabalhadores em busca de emprego em pequenos centros urbanos, utilizando-se metodologia e ferramental simples e acessíveis em praticamente qualquer cidade lationamericana. 
Dias JCP. Aspectos clínicos, sociais e trabalhistas da doença de Chagas em área endêmica sob controle do Estado de Minas Gerais, Biasil. Revista da Sociedade Brasileira de Medicina Tropical 26:93-99, abr-jun, 1993.

\section{NATERIAL E MÉTODOS}

Foram analisados 301 indivíduos que compareceram espontaneamente ao Centro de Pesquisas Emmanuel Dias, em Bambuí, MG, Brasil, entre 1984 e 1986, em busca de exame abreugráfico de tórax como pré-requisito para fichamento em empregos de construção civil na região. Além da abreugrafia tradicional em incidência pósteroanterior $(70 \mathrm{~mm})$, o candidato era submetido a: 1 . sorologia dupla para doença de Chagas, pelas técnicas de hemaglutinação indireta $(1: 16)$ e imunofluorescência indireta $(1: 20)$, processadas no Laboratório de Parasitologia da Fundação Ezequiel Dias, em Belo Horizonte; 2. eletrocardiografia convencional em repouso nas 12 derivações padrão, sendo $\mathrm{D} 2$ repetida por mais 10 complexos ao final do exame, caso extra-sístoles fossem observadas ao longo do traçado de base. Os eletrocardiogramas foram lidos pelo autor, conforme os critérios de Laranja e cols ${ }^{14}$ e a sistematização de Faria ${ }^{10}$, sendo alguns, em caso de dúvida, revistos gentilmente pelo Prof. Anis Rassi, da Universidade de Goiás; 3. abreugrafia contrastada de esôfago em posição oblíquo-anterior-direita, tomada 60 segundos após a ingestão de $150 \mathrm{ml}$ de contraste baritado (técnica de Haddad $€$ Godoy $^{12}$ ) e interpretada pelo autor segundo os critérios cie Rezende ${ }^{15}$; 4 . pequena ficha epidemiológica com ênfase nos antecedentes familiares e doação-recepção de sangue, tomada pelos funcionários do Centro, de longa data acostumados a este tipo de regisiro.

Todos os 301 candidatos eram do sexo masculino e de padrāo sócio-econômico similar entre si, com idades de 18 a 57 anos (mediana de 23 anos), sendo 199 indivíduos da faixa etária 18-29 $\operatorname{anos}(66,1 \%), 69$ da faixa $30-39$ anos $(22,9 \%)$ e 20 da faixa $40-57$ anos (11,0\%). A imensa maioria se compunha de pessoas de origem rural originárias do próprio município de Bambuí, ou de municípios limítrofes.

A análise estatística féz-se pelo qui-quadrado corrigido ou, quando pertinente, pelo teste exato de Fisher, processados pelo programa "Statcalc", do pacote "Epi-Info 5" (PAHO/WHO/CDC-USA), para computador, assim como o cálculo do tamanho da amostra.

Os exames foram gratuitos e consentidos, sendo os resultados entregues aos candidatos em folha própria. Aqueles pacientes com doença de Chagas foram matriculados no Centro Emmanuel Dias, passando a receber atenção médica de rotina. Os não-chagásicos com alterações eletrocardiográficas ou radiológicas importantes foram encaminhados aos serviços de atenção primária à saúde existentes em Bambuí.

\section{RESULTADOS}

Apresentaram anticorpos anti- $T$. cruzi 35 indivíduos examinados, com sorologia concordante, representando uma prevalência de $11,6 \%$ de infecção chagásica nesta amostra. Por grupo etário, a prevalência ficou em 5,0\% dos 18 aos 29 anos, em $13,7 \%$ dos 30 aos 39 e em $39,4 \%$ acima dos 40 anos de idade. Adistribuição etária em cada grupo mostrou uma concentração maior de não-chagásicos no grupo mais jovem $(71,1 \%$ dos 18 aos 29 anos, contra $21,4 \%$ de 30 a 39 e $7,5 \%$ de 40 anos ou acima), distribuindo-se os chagásicos de maneira reversa $(28,5 \%, 34,3 \%$ e $37,1 \%$ respectivamente).

Não houve diferença significativa entre os dois grupos quanto aos antecedentes de vida em zona rural $(88,6 \%$ para os chagásicos e $77,1 \%$ para os não-chagásicos), embora a mediana do tempo vivido em zona rural tivesse sido significativamente maior no grupo soro-positivo (19 anos contra 10 anos).

O grupo soro-positivo apresentou uma proporção significativamente maior de indivíduos com antecedentes imediatos de trabalho na lavoura $(43,8 \%)$ do que os soro-negativos $(19,5 \%$, $\mathrm{p}=0,011)$. Igualmente, foram mais freqüentes os chagásicos que não fizeram ou terminaram o curso primário $(48,6 \%$, contra $19,5 \%$ dos não-chagásicos, $\mathrm{p}=0,0002$ ).

Não houve diferença estatisticamente significativa entre os dois grupos no tocante à informação de parentes com doença de Chagas $(71,4 \%$ de afirmações positivas entre os soropositivos frente a $60,9 \%$ entre os soro-negativos, $\mathrm{p}=0,2275$ ). Também não houve diferenças quanto à referência de morte súbita na família $(57,1 \%$ no grupo chagásico contra $55,3 \%$ no não-chagásico, $\mathrm{p}=0,1939$ ).

Quanto ao conhecimento do triatomíneo, o grupo chagásico apresentou maior proporção de respostas afirmativas $(80,0 \%)$ que o grupo não- 
chagásico $(60,5 \%, \mathrm{p}=0,039)$.

Analisando-se as informações acima, frente aos grupos etários, verificou-se que houve uma tendência crescente de referência à morte súbita na família com o aumentar da idade na amostra como um todo, sem diferenças particulares quanto ao resultado sorológico: $40,2 \%$ para $18-29$ anos, $52,2 \%$ para $30-39$ e $75,8 \%$ para 40 anos ou mais. Igual observação deu-se para o conhecimento do triatomíneo, com percentagens de $56,3 \%, 71,0 \%$ e $84,8 \%$, respectivamente.

Doação de sangue foi referida por 3 pacientes soro-positivos $(8,6 \%)$ e 23 soro-negativos $(8,6 \%)$. Informaram ter recebido sangue 1 chagásico $(2,9 \%)$ e 9 não-chagásicos $(3,4 \%)$, diferença esta não significativa $(p=0,6724)$.

A Tabela 1 resume o panorama eletrocardiográfico para os diferentes grupos etários frente ao resultado sorológico. No seu conjunto, os dados indicam uma ocorrência significativamente maior de eletrocardiogramas alterados no grupo soro-positivo $(31,4 \%)$ que no grupo soro-negativo $(6,8 \%, p=0,0002)$. Por grupo etário, no entanto, diferença significativa só se encontra na faixa de 40 anos ou mais $(p=0,0091)$. A Tabela 2 explicita os achados eletrocardiográficos por grupo etário, entre chagásicos e não-chagásicos. Verifica-se que alterações de maior gravidade e especificidade apenas ocorrem no grupo etário mais elevado, como prenunciado na Tabela 1 .

Quanto à radiologia da área cardíaca, e do esôfago, os dados são sumariados na Tabela 3. De modo geral, os aumentos encontrados da silhueta cardíaca foram discretos em todos os pacientes. Em apenas 2 deles, do grupo soro-positivo mais idoso, detectou-se aumento global moderado. Analogamente, as alterações de esôfago foram todas do grau I de Rezende ${ }^{15}$ no grupo soro-negativo (*), encontrando-se 2 pacientes no grupo soro-positivo com esofagopatia do grau II, um deles com $34 \mathrm{e}$ outro com 45 anos de idade. No cômputo geral, observou-se maior proporção de cardiomegalias no grupo soro-positivo $(12,9 \%)$ frente ao soro-negativo $(1,1 \%, p=0,004)$, o mesmo ocorrendo em relação aos distúrbios esofagianos $(8,6 \%$ e $0,75 \%$, $\mathrm{p}=0,012)$.

Tabela 1 - Resultados de eletrocardiogramas entre trabalhadores chagásicos e não-chagásicos em Bambut, MG, Brasil, por grupo etário.

\begin{tabular}{ccccc}
\hline $\begin{array}{c}\text { Grupo etário } \\
\text { (anos) }\end{array}$ & Eletrocardiogramas & Chagásicos & Não-chagásicos & Totais \\
\hline $18-29$ & normais & 9 & 179 & 188 \\
& alterados & 1 & 10 & 11 \\
$30-39$ & ntermais & 9 & 51 & 60 \\
& alterados & 3 & 6 & 9 \\
$40 \mathrm{e}+$ & normais & 6 & 18 & 24 \\
& alterados & 7 & 2 & 9 \\
\hline Tótais & normais & 24 & 248 & 272 \\
\hline
\end{tabular}

(*) Apenas retardo do trânsito, sem dilatação do órgão. 
Dias JCP. Aspectos clínicos, sociais e trabalhistas da doença de Chagas em área endêmica sob controle do Estado de Minas Gerais, Brasil. Revista da Sociedade Brasileira de Medicina Tropical 26:93-99, abr-jun, 1993.

Tabela 2 - Achados eletrocardiográficos entre trabalhadores chagásicos e não-chagásicos da região de Bambui, MG, Brasil, por grupo etário.

\begin{tabular}{|c|c|c|c|}
\hline $\begin{array}{l}\text { Grupo } \\
\text { etário }\end{array}$ & $\begin{array}{l}\text { Achados } \\
\text { eletrocardiográficos }\left(^{*}\right)\end{array}$ & Chagásicos & Não-chagásicos \\
\hline \multirow[t]{5}{*}{$18-29$} & Normais & 9 & 179 \\
\hline & Alteração primária de $\mathrm{T}$ & 0 & 2 \\
\hline & Sobrecarga ventricular esquerda & 0 & 1 \\
\hline & Extra-sístoles ventriculares isoladas & 0 & 3 \\
\hline & bigeminadas & 1 & 4 \\
\hline \multirow[t]{8}{*}{$30-39$} & Normais & 9 & 51 \\
\hline & Bloqueio $A-V$ de primeiro grau & 1 & 0 \\
\hline & Alteração primária de $T$ & 0 & 1 \\
\hline & Sobrecarga ventricular esquerda & 1 & 2 \\
\hline & Extra-sístoles ventr. isoladas & 1 & 2 \\
\hline & Hemibloqueio anterior esquerdo (HBAE) & 1 & 0 \\
\hline & Baixa voltagem de QRS & 3 & 0 \\
\hline & Área hipo ativa ântero-septal & 0 & 1 \\
\hline \multirow[t]{9}{*}{$40 \mathrm{e}+$} & Normais & 6 & 18 \\
\hline & Bloqueio $A-V$ de primeiro grau & 0 & 1 \\
\hline & Bloqueio $\mathrm{A}-\mathrm{V}$ total (intermitente) & 1 & 0 \\
\hline & Alteração primária de $T$ & 1 & 0 \\
\hline & Extra-sístoles ventriculares isoladas & 1 & 0 \\
\hline & bigeminadas & 0 & 1 \\
\hline & Bloq. compl. ramo direito $+\mathrm{HBAE}$ & 3 & 0 \\
\hline & HBAE & 2 & 0 \\
\hline & Baixa voltagem de $Q R S$ & 1 & $\mathbf{0}$ \\
\hline
\end{tabular}

(*) Mais de uma alteração pode estar presente no mesmo traçado

\begin{tabular}{|c|c|c|c|c|c|}
\hline \multirow{2}{*}{$\begin{array}{l}\text { Grupo } \\
\text { etạrio }\end{array}$} & & \multicolumn{2}{|c|}{ Chagásicos } & \multicolumn{2}{|c|}{ Não-ghagásicos } \\
\hline & & área cardíaca & esôfago & área cardíaca & esôfago \\
\hline $18-39$ & $\begin{array}{l}\text { pormais } \\
\text { alterados }\end{array}$ & 18 & 10 & 188 & $\begin{array}{r}188 \\
1\end{array}$ \\
\hline $3 \theta-39$ & $\begin{array}{l}\text { nofmais } \\
\text { alterados }\end{array}$ & 11 & $\begin{array}{l}10 \\
2\end{array}$ & $\begin{array}{r}57 \\
0\end{array}$ & $\begin{array}{r}56 \\
1\end{array}$ \\
\hline $40 e+$ & $\begin{array}{l}\text { normais } \\
\text { alterados }\end{array}$ & $\begin{array}{r}10 \\
3\end{array}$ & $\begin{array}{r}12 \\
1\end{array}$ & $\begin{array}{r}18 \\
2\end{array}$ & $\begin{array}{r}2 \beta \\
0\end{array}$ \\
\hline Totais & $\begin{array}{l}\text { normais } \\
\text { alterados }\end{array}$ & $\begin{array}{r}31 \\
4\end{array}$ & $\begin{array}{r}32 \\
3\end{array}$ & $\begin{array}{r}263 \\
3\end{array}$ & $\begin{array}{r}264 \\
2\end{array}$ \\
\hline
\end{tabular}

* Técnica de Haddad \& Godoy (11). 


\section{DISCUSSÃO}

A região do Oeste de Minas Gerais onde se situa o Município de Bambuí foi extremamente afetada pela doença de Chagas humana em décadas anteriores a 1960, alcançando-se facilmente taxas gerais de prevalência ao redor de 40 a $50 \%$ da população geral não-selecionada, havendo sub-regiões onde aos 10 anos de idade $45 \%$ das crianças já apresentavam anticorpos anti-T. cruzi $^{113}$.

A queda da prevalência da infecção chagásica em Bambuí, após o controle efetivo da transmissão vetorial, fora prevista por Emmanuel Dias já em $1957^{3}$ e observada através de inquéritos soroepidemiológicos em 1965, 1974 e $1980^{4}$, em paralelo ao que se verificava no Estado de São Paulo a partir de $1968^{18} 20$. Implantada efetiva vigilância epidemiológica contra os triatomíneos domiciliados já em 1974, a transmissão da doença de Chagas humana praticamente desapareceu em Bambuí, observando-se em paralelo um bom controle das relativamente poucas transfusões de sangue ali realizadas anualmente $e$, indubitavelmente, uma expressão mínima da transmissão congênita na Região ${ }^{5}$. Assim, para a década de 80 , o encontro de $11,6 \%$ da protozoose entre homens adultos da área, em sua imensa maioria originários da zona rural, reflete fielmente tanto a situação pré-profilática como o impacto muito forte do programa de controle, ambos claramente expressos nos resultados sorológicos por grupo etário. O mesmo padrão ocorreu em Iguatama e Pains, municípios vizinhos, em 1983'. Quantoà representatividade dos presentes dados, a amostra foi estatisticamente satisfatória (**). Resta analisar se a demanda espontânea de pessoas em busca de trabalho não pré-selecionaria indivíduos mais sadios, marginalizando os chagásicos com cardiopatia avançada, situação que tem ocorrido em investigações semelhantes, como a de Faria ${ }^{10}$. Indubitavelmente, no entanto, o dado reflete o panorama do grupo social objeto do trabalho, ou seja, trabalhadores de uma área endêmica de doença de Chagas. Neste sentido, as informações colhidas na anamnese sumária retratam

(**) Para a população de 12.000 indivíduos na faixa etária apreciada no Município, uma prevalência de $14 \%$ e um desvio máximo de $10 \%$, a amostra seria de 282 pessoas [i.c. $=95 \%$ (Epi Info)]. a realidade vigente na área pesquisada, mostrando o gradual deslocamento dos lavradores para as periferias urbanas e para atividades mais típicas do modelo urbano-industrial, como assinalado em outras publicações $^{111} 19$.

A maior permanência dos indivíduos soropositivos em áreas rurais e o maior conhecimento do triatomíneo reforçam a importância que teve a transmissão vetorial do $T$. cruzi na região, como observado recentemente no Estado de Goiás ${ }^{23}$.

Por seu turno, antecedentes imediatos de trabalho na lavoura e de menor escolaridade foram significativamente mais referidos pelo grupo de infectados, associando o chagásico aos extratos mais primários de produção ${ }^{819}$. Já os antecedentes familiares de casos da doença e morte súbita, significativamente mais presentes entre os entrevistados mais velhos, espelham a alta prevalência e o importante grau de morbi-mortalidade da endemia na região em épocas passadas, como referido em clássicos trabalhos da década de $50^{213}$ 14. Por outro lado, os dados em conjunto vão indicando que paulatinamente o impacto médico social da doença vai sendo reduzido na área, conforme o vaticínio de Emmanuel Dias e certamente em função do trabalho profilático ali realizado ${ }^{3} 17$.

Doação e recepção de sangue foram igual e escassamente referidas nos dois grupos estudados, particularmente a recepção. Em áreas rurais e pequenos municípios brasileiros, a prática da hemoterapia é ainda discreta, conforme dados recentes, embora sempre se encontrem pessoas chagásicas que mencionem já ter doado sangue ${ }^{8}$. De qualquer forma, os dados em conjunto sugerem que a transmissão transfusional do $T$. cruzi seja pouco importante na área em apreço.

O estudo da morbidade da doença de Chagas em indivíduos adultos, que estão em atividade laboral, apresenta grande importância na medida em que 16 milhões de latinoamericanos são portadores da infecção e, em sua maioria, dependem de trabalho físico para sobreviver ${ }^{11171922}$. A exemplo de outros estudos, os dados da presente investigação demonstram a ocorrência na região de Bambuí, em anos recentes, de ainda significativa proporção de indivíduos economicamente ativos com cardiopatia chagásica crônica, algo próximo a $5 \%$ da população total na faixa de 17 a 57 anos de idade. Como acima assinalado, esta proporção pode ser ainda maior, se 
Dias JCP. Aspectos clínicos, sociais e trabalhistas da doença de Chagas em área endêmica sob controle do Estado de Minas Gerais, Brasil. Revista da Sociedade Brasileira de Medicina Tropical 26:93-99, abr-jun, 1993.

considerados aqueles indivíduos que não se apresentaram à seleção laboral justamente em face de sua incapacidade física. Tudo isso ainda reflete a importância da infecção chagásica na área, muito mais acentuada em décadas passadas (com cifras próximas aos $20 \%$ de cardiopatia chagásica entre a população adulta do Município ${ }^{13}$.

Não há dúvida de que a doença de Chagas crônica limita o trabalho e a vida de trabalhadores brasileiros, seja na zona rural como em $\mathrm{Luz}^{10}$ ou Iguatama $^{1}$, seja em cidades como Brasília ${ }^{21}$ ou São Paulo $^{11}$. No entanto, em sua maioria são indivíduos em forma indeterminada ou com graus iniciais de comprometimento miocárdico, demonstrável apenas por alterações leves ou moderadas do eletrocardiograma não associadas com insuficiência cardíaca e/ou cardiomegalias ${ }^{8}$. Do ponto de vista laboral, em sua imensa maioria se igualam no desempenho físico os chagásicos e não-chagásicos mais jovens, mormente quando é normal o eletrocardiograma ${ }^{10}$. Já no grupo etário mais elevado, as alterações eletrocardiográficas mais características da esquizotripanose $e o$ aparecimento de cardiomegalias mais acentuadas indicam o caráter evolutivo da doença ${ }^{5} 71014$. É este um desafio complementar às autoridades sanitárias brasileiras, que têm conseguido interromper a transmissão vetorial da doença em vastas extensões do País: prover atenção médica e previdenciária adequada para cerca de 5 milhões de infectados são produto da situação epidemiológica passada ${ }^{6}{ }^{17}$.

Quanto à esofagopatia chagásica, os presentes dados reforçam outros mais antigos de Bambur e de outras áreas endêmicas brasileiras, com prevalência da disperistalse ao redor de $8 \%$ dos infectados, em sua maioria no grupo I de Rezende na faixa etária considerada $^{1915}$.

Em seu conjunto, o trabalho demonstra uma vez mais o impacto positivo dos programas de controle do vetor sobre a prevalência e a morbidade da doença de Chagas numa área endêmica. Em termos de Brasil, tal observação reforça a enorme necessidade de que se mantenha o programa governamental $^{17} 22$. Demonstra ainda que com instrumental simples podem ser detectadas formas iniciais da cardiopatia e esofagopatia chagásicas, como elemento de real benefício à população infectada ${ }^{7}$. Mostra finalmente que embora limitantes a longo prazo, as principais formas clínicas da esquizotripanose crônica são suportadas pelo chagásico em suas etapas iniciais, sendo mais importantes para ele as oportunidades de trabalho que a doença porventura existente.

\section{SUMMARY}

Among 301 construction workers examined in Bambui, Brasil, $11.6 \%$ presented positive serology for American trypanosomiasis, with a prevalence of $5.0 \%$ in the age group 17-29 years, $13.8 \%$ in the 30-39 and $39.4 \%$ in the $40-57$. Electrocardiographic disturbances were detected in $31.3 \%$ of the seropositive and in $7.1 \%$ of the seronegative individuals, with a significant difference in the age group $40-57$ years. Cardiac enlargement and oesophagopathy were detected respectively in $8.6 \%$ and $8.6 \%$ of the positive and $1.5 \%$ and $0.8 \%$ of the negative group. Chagasic and nonchagasic individuals came from the same region and social status, but the seropositive group was more associated with low scholarship and immediate antecedent of rural activities. The effectiveness of the programme of vector control installed in the Region in the years 1970 is once more confirmed by its positive impact on the prevalence and morbidity of human Chagas disease. Congenital and transfusional Chagas disease seem to play a very small epidemiologic role in the Region at the present time. Control.

Key-words: Chagas disease. Work. Morbidity.

\section{AGRADECIMENTOS}

Agradecemos ao Laboratório de Parasitologia da Fundação Ezequiel Dias, na pessoa da Dra. Eliana Furtado Moreira, pelos exames sorológicos realizados.

\section{REFERÊNCIAS BIBLIOGRÁFICAS}

1. Coura JR, Abreu LL, Dubois LEG, Lima FC, Arruda JrE, Willcox HPF, Anunziato N, Petana W. Morbidade da doença de Chagas. II - Estudos seccionais em quatro áreas de campo no Brasil. Memórias do Instituto Oswaldo Cruz 79:101-124, 1984.

2. Dias E. Doença de Chagas, um problema americano. Hospital 55:57-65, 1959.

3. Dias E. Profilaxia da doença de Chagas. Jornal Brasileiro de Medicina 1:939-954, 1959.

4. Dias JCP. Prevalência da doença de Chagas entre crianças da zona rural de Bambuí, MG, após ensaio 
profilático. Revista Brasileira de Malariologia e Doenças Tropicais 19:135-159, 1967.

5. Dias JCP. Doença de Chagas em Bambuí, Minas Gerais, Brasil. Estudo clínico e epidemiológico a partir da fase aguda, entre 1940 e 1982 . Tese. Faculdade de Medicina da Universidade Federal de Minas Gerais, Belo Horizonte, 1982.

6. Dias JCP. Control of Chagas disease in Brazil. Parasitology Today 3:336-341, 1987.

7. Dias JCP. Doença de Chagas: Clínica e Terapêutica. SUCAM, Ministério da Saúde, Brasília, 1989.

8. Dias JCP. Epidemiology of Chagas disease. In: Wendel S, Brener Z, Camargo ME, Rassi A (eds) Chagas disease (American trypanosomiasis): its impact on transfusion and clinical medicine. Sociedade Brasileira de Hematologia e Hemoterapia, São Paulo p.49-80, 1992.

9. Dias JCP, Camacho LAB, Silva JC, Magalhães JS, Krieger $\mathbf{H}$. Esofagopatia chagásica na área endêmica de Bambuí, MG, Brasil. Revista da Sociedade Brasileira de Medicina Tropical 16:46-57, 1983.

10. Faria CAF. Ergometria na avaliação clínica da doença de Chagas crônica. In: Cançado JR, Chuster M (eds) Cardiopatia chagásica, Fundação Carlos Chagas, Belo Horizonte p.223-254, 1985.

11. Goldbaum M. Saúde e Trabalho. A doença de Chagas no setor industrial. Tese. Faculdade de Medicina da Universidade de São Paulo, São Paulo, 1981.

12. Haddad N, Godoy RA. Valor da medida do tempo de trânsito esofágico como meio de diagnóstico precoce da disperistalse de esôfago em indivíduos chagásicos. Revista Goiana de Medicina 9:45-60, 1963.

13. Laranja FS, Dias E, Duarte E, Pellegrino J. Observações clínicas e epidemiológicas sobre a moléstia de Chagas no Oeste de Minas Gerais.
Hospital 40:945-988, 1951

14. Laranja FS, Dias E, Nóbrega GC, Miranda A. Chagas' disease: a clinical, epidemiologic and pathologic study. Circulation 14:1035-1060, 1956.

15. Rézende JM. Clínica: manifestações digestivas. In: Brener Z, Andrade Z (eds) Trypanosoma cruzi e doença de Chagas. Guanabara Koogan, Rio de Janeiro p.313-361, 1979.

16. Schmuñiz GA. Trypanosoma cruzi, the etiologic agent of Chagas disease: status in the supply in endemic and nonendemic countries. Transfusion 31:547-557, 1991

17. Schofield CJ, Dias JCP. A cost-benefit analysis of Chagas disease control. Memórias do Instituto Oswaldo Cruz 86:285-295, 1991.

18. Rocha e Silva EO. Profilaxia. In: Brener Z, Andrade $\mathrm{Z}$ (eds) Trypanosoma cruzi e doença de Chagas. Guanabara Koogan, Rio de Janeiro p.425-449, 1970.

19. Silva GR, Litvoc J, Goldbaum M, Dias JCP. Aspectos da epidemiologia da doença de Chagas. Ciência e Cultura 31(supl.):81-103, 1979.

20. Souza AG, Wanderley DMV, Buralli GM, Andrade JCR. Consolidation of the control of Chagas' disease vectors in the state of São Paulo. Memórias do Instituto Oswaldo Cruz 79(supl.): 125-132, 1984.

21. Tinoco DL. Prevalência da doença de Chagas. Confirmação do diagnóstico pelo immunoblot. Tese. Universidade de Brasília, Brasília, 1982.

22. World Health Organization. Control of Chagas disease. Report of a WHO Expert Committee. Geneva. WHO Technical Report Series 811, 1991.

23. Zicker F, Oliveira RM, Luquetti AO, Oliveira OS, Smith PG. Seroprevalence of Trypanosoma cruzi infection among unskilled urban workers in central Brazil. Transactions of the Royal Society of Tropical Medicine and Hygiene 83:511-513, 1989. 